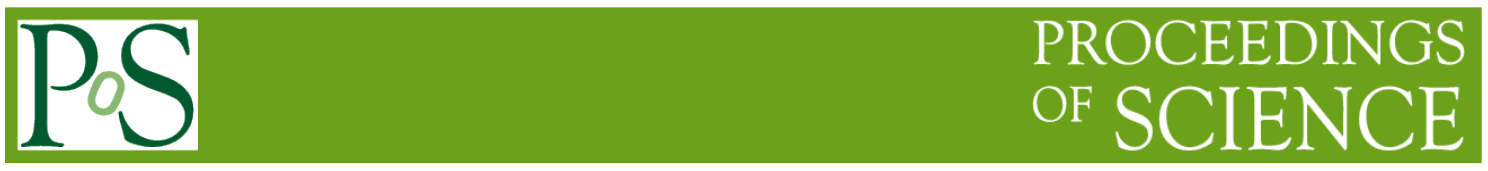

\title{
iThEC ADS Initiatives
}

\author{
Maurice Bourquin ${ }^{1}$ \\ University of Geneva \\ 1205 Geneva, Switzerland \\ E-mail: maurice.bourquineunige.ch
}

\section{On behalf of the international Thorium Energy Committee (iThEC) \\ Rue François-Dussaud 17 \\ 1227 Geneva, Switzerland}

Web : http://ithec.org/en/organisation

The international Thorium Energy Committee (iThEC), a not for profit association, under Swiss law, was founded in 2012. Its members are engineers, physicists, and other concerned citizens acting to promote $\mathrm{R} \& \mathrm{D}$ on the use of thorium in order to transmute nuclear waste and produce safe, clean and abundant energy. To meet the tremendous world energy needs, systematic R\&D has to be pursued to replace fossil fuels. Nuclear energy, which produces no green-house gases and no air pollution, should be a leading candidate, and in this context, thorium represents a great potential. iThEC is at the origin of two new initiatives, which I will present: a first ADS experiment of substantial power ( $\geq 1 \mathrm{MW}$ ) and a high-power superconducting cyclotron design with related prototypes. Global cooperation is highly desirable in this domain, and I will discuss possibilities of cooperation based on the CERN experiment model.

4th Workshop on ADS and thorium

31 August - 2 September 2016

University of Huddersfield, England

\footnotetext{
${ }^{1}$ Speaker
} 


\section{Introduction}

We live in a world where the demand for electricity is increasing and where the use of fossil fuels is threatening the environment. The massive earthquake and the subsequent tsunami that hit northern Japan in 2011 and the related nuclear accidents at Fukushima (as well as the Chernobyl accident) have triggered a profound reflection about the future of the commercial nuclear energy sector, and the whole energy sector.

This is also the case in Switzerland, where the electricity mix included in $201454.2 \%$ of hydroelectricity, $39.1 \%$ of nuclear, $5.7 \%$ of fossil and negligible amounts of solar and wind power. For a total of $56 \mathrm{TWh}$ consumed, $61.5 \mathrm{TWh}$ were produced in the country.

One should also note that on the commercial front about 40 TWh have been imported and a similar amount has been exported to neighboring countries. These facts show the economic attractivity for Switzerland of the European wholesale market. It puts the Swiss hydroelectric and nuclear installations under pressure, as they will not anymore cover their costs. One is confronted with the question of whether or not political measures should be taken to minimize induced economic biases.

In the weeks following the nuclear accident at Fukushima, the Swiss government indicated to the parliament that nuclear power in Switzerland will be phased out. The five plants in operation would not be replaced. An "Energy Strategy 2050" would be put in place, including enhancing energy efficiency, developing renewable energy production (increase of hydropower by 3.2 TWh), developing new renewable energy (increase of solar, wind and biomass by $24.2 \mathrm{TWh}$ ), meeting residual demand through fossil fuels and electricity imports, expanding and renovating the electricity networks, strengthening research and encouraging international cooperation in the field of energy.

Even if nuclear energy is phased-out, Switzerland, as the rest of the world, will still need to address the safe, economic and definitive management of all the radioactive waste produced in the last 60 years. For example the cumulative amount of spent fuel discharged from 1990 to 2030 will reach $600 \times 1000$ tons of heavy metal (tHM) according to data from IAEA-TECDOC1613, April 2009. The long-term plan for high-level wastes in Switzerland is to construct deep geological repositories in two sites. As in many other countries, such a plan presents major difficulties in its technological feasibility and in the concern by the population.

One is thus led to address the following questions: is there an alternative to the uranium-fueled nuclear power plants? Can the amount and the lifetimes of radioactive wastes be reduced? Can one make nuclear energy acceptable to Society?

\section{The international Thorium Energy Committee}

Those questions are at the basis of the activities of iThEC, an association aiming at developing thorium energy systems, with a focus on Accelerator-Driven Systems (ADS). The international 
Thorium Energy Committee is a not for profit association, under Swiss law, founded in 2012. Its members include physicists, engineers, scholars, politicians and other concerned citizens acting to promote $\mathrm{R} \& \mathrm{D}$ and the use of thorium in order to transmute long-lived nuclear waste and produce safe, clean and abundant energy sources, in particular in view of the expected huge energy needs of developing countries.

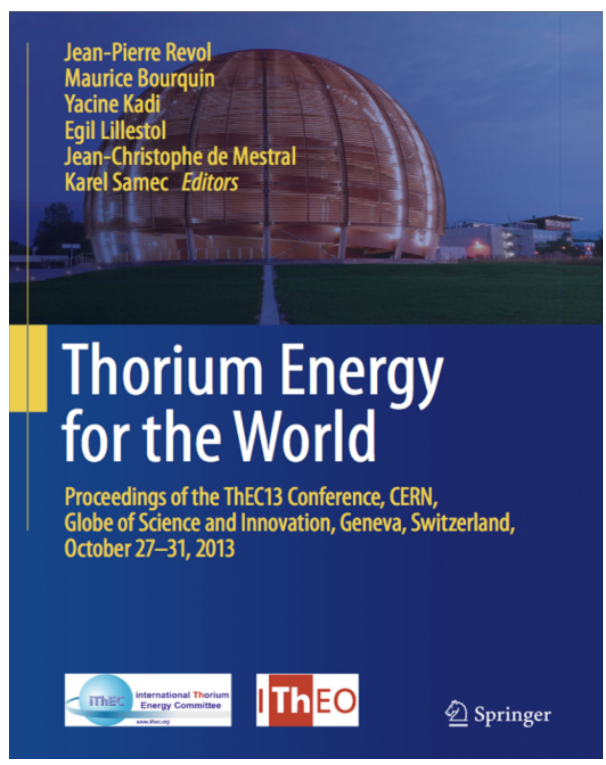
fissile. This gives a potential gain factor of 140 compared to ${ }^{235} \mathrm{U}$ in PWR (in addition to the factor of 4 in abundance).

${ }^{233} \mathrm{U}$ is an excellent fuel for a breeder system, especially with fast neutrons, where the number of neutrons produced with respect to neutrons absorbed is about 2.3 . But the environment $\left({ }^{232} \mathrm{Th}\right.$, ${ }^{238} \mathrm{U}$ ) has to be taken into account. ${ }^{232} \mathrm{Th}$ and ${ }^{233} \mathrm{U}$ cannot simply be substituted to PWR fuel, mainly because of the higher neutron capture rate on thorium and the relatively long half-life of ${ }^{233} \mathrm{~Pa}$.

What are the reactor options for using thorium? One can distinguish three cases: 1. Use thorium blankets around reactors to breed ${ }^{233} \mathrm{U}$ and introduce ${ }^{233} \mathrm{U}$ in the fuel $\left(\mathrm{n}+{ }^{232} \mathrm{Th}{ }_{\mathrm{Fr}}{ }^{233} \mathrm{Th}\right.$

$\left.{ }^{23}{ }^{233} \mathrm{~Pa}{ }_{\text {تᄑ }}{ }^{233} \mathrm{U}\right) ; 2$. Continuously move the fuel out, in order to always have fresh fuel such as in pebble bed reactors (once through) or in molten salt reactors (reprocessing on-line); 3. Provide an external neutron source, an Accelerator Driven Systems: this is the solution proposed by Carlo Rubbia at CERN in the 1990's (ref. 2) and promoted by iThEC. The ADS is a subcritical approach, where a particle accelerator provides an external neutron source through spallation, a core in which both source neutrons and fission neutrons are at work with a moderator allowing for a fast neutron spectrum.

At the ThEC13 conference, Stanislav Sidorkin presented a proposal for an ADS experiment at Troitsk, using the Moscow Meson Factory, at the Institute for Nuclear Research (INR). iThEC recognized that it could be a realistic possibility of having a new step forward in ADS. One would couple a proton beam to a subcritical core for the first time at significant power $(\geq 1$ MW) to characterise the properties of ADS, demonstrate safety and learn how to operate such a system. This would provide invaluable input for designing and constructing an industrial prototype, as well as developing a future thorium fuel cycle. 
To define the parameters of the project, some guidelines have to be followed. Concerning safety: eliminate criticality accidents by making the system subcritical, operate the system with passive safety elements to avoid core melting or limit its consequences borrowing features from US advanced fast critical reactor designs, avoid dangerous coolants such as liquid sodium. Concerning waste management: use fast neutrons, thorium fuel, and recycle long-lived transuranic actinides (TRU) to minimize waste. Concerning military proliferation: use thorium fuel (small Pu prod., ${ }^{233} \mathrm{U}$ a very difficult mixture to manufacture weapons), avoid $\mathrm{Pu}$ separation (Purex), use pyroelectro reprocessing instead (developed for uranium at Argonne National Lab, USA).

\section{The INR/iThEC ADS Project at Troitsk}

iThEC is considering using an existing facility located at the Institute of Nuclear Research at Troitsk near Moscow. The existing INR facility includes a proton LINAC designed to reach an energy of $600 \mathrm{MeV}$ with a power of $300 \mathrm{~kW}$, a spallation neutron source, a pit on a beam line to receive a subcritical core, and the necessary infrastructure (shielding, handling devices) to manipulate highly radioactive material.

An ADS experiment can be implemented with the following goals:

1) Measure the physical properties of a 1 to 3 MW ADS (coupling of the accelerator to different core configurations; demonstrate safety; develop operational procedures).

2) Demonstrate transmutation of nuclear waste (MA \& LLFP/TARC).

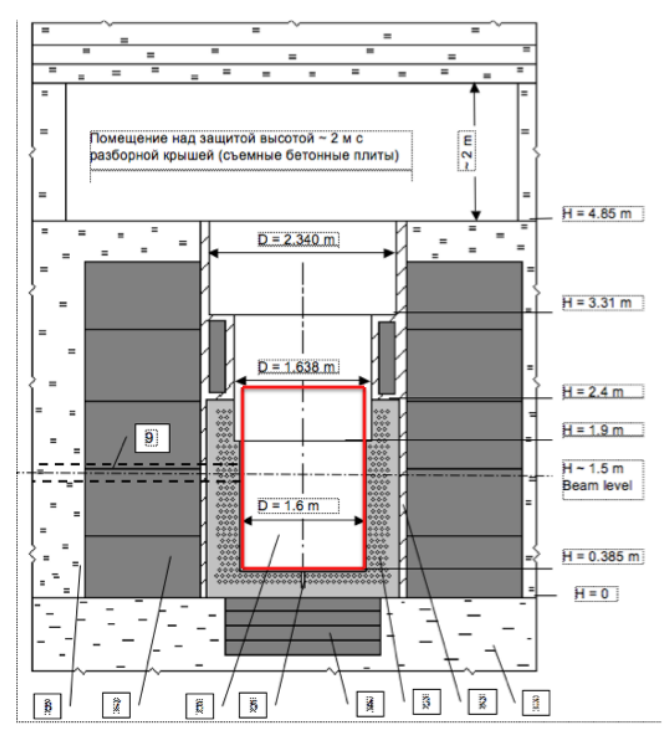

Thanks to the availability of the facility, the experiment would be faster and cheaper than current projects. The pit to be used for the target and the core is shown here, with the reflector already in place.

The main principles of the core concept are to be designed and submitted for approval to Russian safety authorities by ROSATOM's specialized agencies. They are: a fast neutron flux similar to a fast reactor flux $\left(10^{14} \mathrm{n} / \mathrm{cm}^{2} / \mathrm{s}\right)$; a minimum inventory of fuel; a thermal drive zone, but a fast driver zone will also be studied; a simplified cooling system and control of reactivity, which should result in a significant cost reduction.

The control and protection system, as well as the physical and technical parameters of the ADS must comply with the technical regulations of the Russian Federation. 


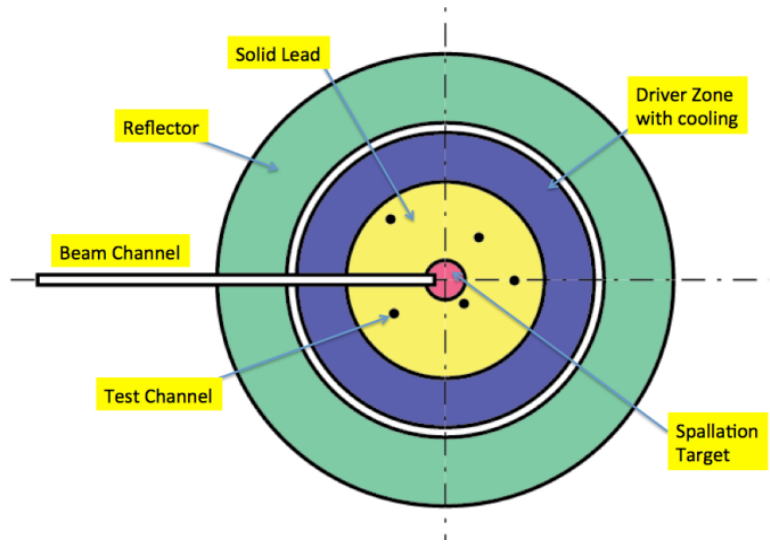

The technical assumptions include:

and assuming $\mathrm{G}_{0}=1.15$, which will have to be refined for the specific target to be used;

- In order to guarantee that a thermal power of $1 \mathrm{MW}$ can be reached, $\mathrm{k}$ will have to reach 0.972 , and in general during operation $\mathrm{k}$ will be varied up to a maximum value of 0.98 , to characterize the core to accelerator coupling over the largest $\mathrm{k}$ range;

- As the beam power is relatively small, the target will be a solid target, most likely tungsten, water-cooled. This is a simplification and a significant saving on the cost;

- As the the maximum thermal power could reach $3 \mathrm{MW}$ (at $75 \mathrm{~kW}$ beam power), the cooling of the core should allow for this.

The scientific program includes: mapping of the neutron flux (energy and intensity) inside the lead volume; start-up and shut down procedures; precision measurement of reactivity and monitoring of the time evolution of the reactivity; operation and monitoring of the system at steady state; practical coupling of the accelerator, the spallation target and the subcritical core, reaction to change in beam power; measurements of the temperature coefficient.

In a letter to the President of iThEC dated May 26, 2016, the Director of INR is indicating that "INR is considering this proposal very seriously and it has a high priority in the Institute research program". In this context it is worthwhile to quote a recent announcement from the Russian government: "Russia's president Vladimir Putin has asked state nuclear corporation Rosatom and the Kurchatov Institute national research center to prepare a proposal by 1 March 2017 on the prospects for using thorium for nuclear fuel".

INR and iThEC have jointly prepared a roadmap for the next 5 years, which includes: 0 Preliminary phase (6 months): specifications of technical parameters; 1 - Conceptual design; 2Obtaining government authorization, which requires three steps: declaration of intent, estimate of the impact on the environment, funding plan; 3- Technical design; 4- Licensing; 5Permission for construction and installation; 6- Construction and installation; 7-Permission for commissioning and operation.

In summary the project will be an experiment to study the coupling of an accelerator to a subcritical core at significant power ( $\geq 1 \mathrm{MW}$ thermal), as ADS is the most practical way of using thorium, for nuclear waste transmutation and for energy production; a unique strategic development toward thorium technologies applied to the energy domain, based on an existing facility; an input for the design of an industrial ADS. 
INR Moscow, in cooperation with iThEC, with other institutes in Russia and abroad are in the process to form an international collaboration.

\section{The iThEC Superconducting High-Power Cyclotron Project}

What are the main requirements for an ADS accelerator? In principle, it does not matter how the external neutron source is provided. However, in practice, for industrial applications, there are many requirements which make the accelerator challenging. The proton beam energy should ideally be above $900 \mathrm{MeV}$, but a lower energy can be compensated by a higher current. The beam power should reach a value between a few MW to about $10 \mathrm{MW}$, depending on the required application. A large operational range is desirable to adapt the beam power to the operation of the reactor and the fluctuating electric power demand associated with the network. The size of the beam spot of the target entrance window has a large impact, following studies conducted at JAEA and MYRRHA. Beam losses have to be tightly controlled to minimize irradiation of the accelerator and of the environment: a figure of merit for LINAC's is less than $1 \mathrm{~W} / \mathrm{m}$, while for cyclotrons losses are mainly localized at injection and extraction.

The issue of reliability requiring minimizing beam trips is a significant challenge: the limitation mainly comes from thermal stress inducing fatigue in beam window, fuel cladding and vessel structure.

Solutions include making the accelerator more reliable, adding redundancy (several sources, several accelerators, etc.), improvement in materials, maintenance and operation, relaxing the demands from reactors with a Molten Salt-ADS, etc.

At the ThEC13 conference, Pierre Mandrillon from AIMA Development, Lacassagne Cyclotron Laboratory, presented the concept of a new high power superconducting cyclotron, in principle adoptable as a driver for an Accelerator-Driven System (Ref.3): the AIMA Reverse Valley BField Cyclotron ${ }^{\circledR}$.

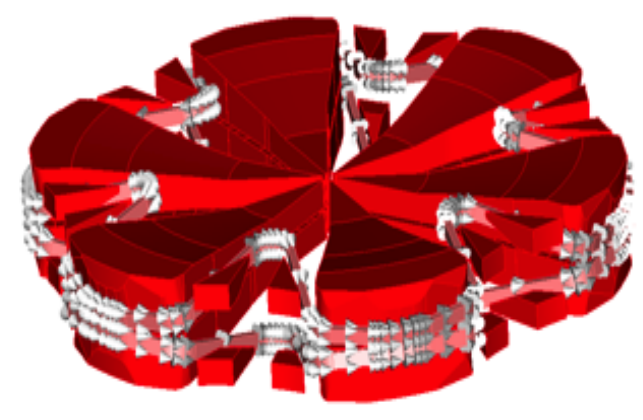

It includes the following features.

High reliability: 3 sources; smaller number of components compared to a linac.

High efficiency: superconductivity reduces ohmic losses.

Reduced Impact on the environment: the beam losses are localized.

A less expensive infrastructure: the gain comes from the much more compact infrastructure compared to a linac.

Estimated lower cost: the overall cost is to be several times less than the cost of an equivalent linac, as it is one of the goals of the iThEC project to evaluate the price.

A major advantage is that the B-field configuration in the central region allows acceleration at low energies; an injector cyclotron is not needed. The proton extraction is performed through stripping of $\mathrm{H}_{2}^{+}$. 
The objective of the project is to deliver an optimized conceptual design of the cyclotron and the related interfaces that is in principle adoptable by the market. We plan to demonstrate the main conceptual aspect of our design with prototypes and proof of concept demonstrators that are aimed to bridge the gap to industrialization. We plan to get a full understanding of the cost drivers that are very important parameters for innovative applications.

This research project will be submitted to the EU-H2020 "Future Emerging Technologies" call, to deliver a conceptual design_for a superconducting one stage $\mathrm{H}_{2}{ }^{+}$cyclotron with 3 injections, with energy and beam currents optimized for the scope of radioactive waste transmutation ( 600 $\mathrm{MeV}, 6 \mathrm{~mA}$ as reference design), and possibly high electrical efficiency ( $>40 \%)$. Immediate diverse possible applications can be envisaged for energy production (ADS) and high flux particle beams. The collaborating institutions are iThEC, the Paul Scherrer Institute, AIMA Development, ASG, Hydromine Nuclear Energy, and Nuclear-21. The project will be coordinated by CERN.

\section{Conclusions}

iThEC has initiated two projects to contribute to the energy problems of tomorrow: 1.the formation of a multi-national collaboration to use the INR-Troitsk facility for ADS; a workshop will be organized next year; we are looking for political and financial support in several countries; 2. an EU-H2020 project, implemented through a Future Emerging Technology call (FET) of designing a single stage High Power Superconducting Cyclotron to drive spallation neutron sources.

Acknowledgments The author would like to thank all the colleagues of iThEC working around these initiatives.

\section{References}

1. Thorium Energy for the World, Proc. of ThEC13 Conf., CERN Globe of Science and Innovation, Geneva, Switzerland, October 27-31, 2013, Publ. by Springer 2016.

2. C. Rubbia et al., Conceptual Design of a Fast Neutron Operated High Power Energy Amplifier, CERN/AT/95--44 (ET) 